\title{
Image Processing Algorithms for Driver Assistance using Wide Angle Cameras
}

\author{
Sebastian Houben* \\ * Institute for Neural Computation, University of Bochum, Germany \\ Advisors: Dr. Gregor Schner and Dr. Rolf Wrtz \\ Date and location of $\mathrm{PhD}$ thesis defense: 20th November 2015, University of Bochum, Germany
}

Received 28st June 2016; accepted 1st August 2016

\begin{abstract}
Modern vehicles are deployed with a large number of sensors in order to provide a rich spectrum of driver assistance functionality. These systems enhance security and comfort of passengers and other traffic participants alike, but they also pave the road to fully autonomous traffic. In order to provide this functionality robustly and reliably, one currently makes use of numerous specialized sensors: laser, radar, ultrasound, and infrared sensors, as well as different kinds of video cameras.

The diversity of sensors comes with high cost and enables complex assistance functions momentarily only for upper-class vehicles. The current research, thus, focuses on the development of better algorithms that make similar systems possible on inexpensive sensors.
\end{abstract}

\section{Detection of motion: Rear-crossing traffic}

One task of video-based systems is the detection of objects in motion in order to anticipate and avoid predictions at the earliest opportunity. In the course of the thesis, we developed a rear-crossing traffic alert system that aims to detect general objects in motion, without any assumptions of the shape or appearance. It was shown that a common optical flow estimator can be combined with the camera motion reconstructed from vehicle odometry in order to recognize crossing objects approximately 2 before a potential collision.

\section{Model-based object detection and recognition: Parking lots and trailers}

Algorithms for recognizing closely specified classes of objects, like vehicles, can be separated into two fundamental groups: model-based algorithms try to estimate very few object parameters from image data, parameterfree algorithms, on the other hand, that are substantially influenced by methods from machine learning aim at extracting characteristic features from data in order to relocalize them later on. The model-based approach

Correspondence to: < sebastian.houben@ini.rub.de>

Recommended for acceptance by David Vázquez Bermúdez

ELCVIA ISSN:1577-5097

DOI:http://dx.doi.org/10.5565/rev/elcvia.943

Published by Computer Vision Center / Universitat Autònoma de Barcelona, Barcelona, Spain 


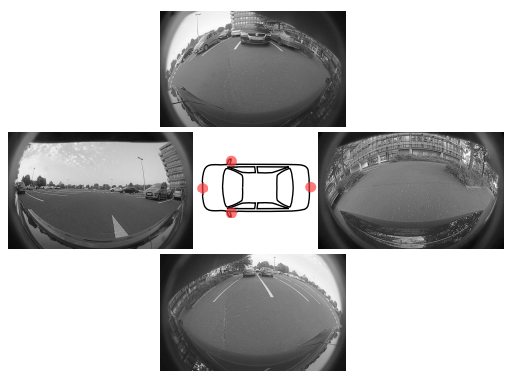

(a)

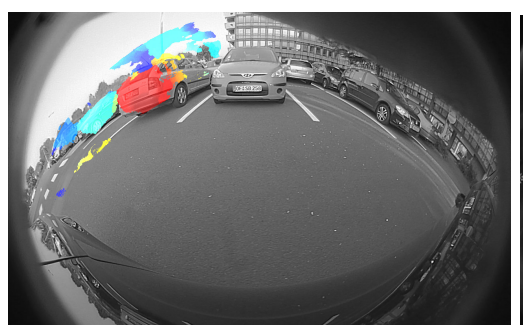

(b)

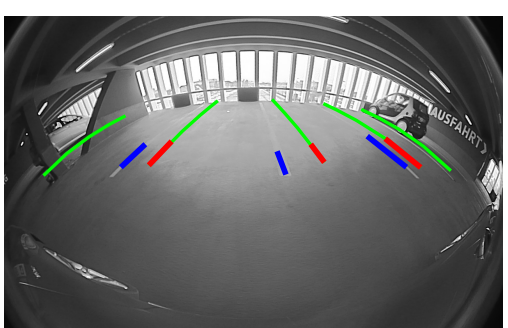

(c)

Figure 1:

(a) The setup at hand consists of four wide-angle cameras providing a view surrounding the entire vehicle.

(b) Image of a side mirror camera with color-coded distance measures retrieved by stereo vision with a front camera.

(c) Image taken in a parking scenario with the known map of park markings overlaid in green and detections in blue.

is demonstrated multiple times within this thesis, e.g., for recognizing park markings [9] and tracking of a trailer coupled to the vehicle [5]. The later was evaluated with the help of a rear-end topview camera where the presence of a trailer as well as the articulation angle with respect to the vehicle longitudinal axis was computed solely based on video data. The average deviation was below $2^{\circ}$ which allows the system to assist the driver during difficult maneuvers, e.g., when driving backwards, and to anticipate dangerous situations due to overswinging that might build up during long drives at higher speed. The parameter-free approach was used for classification of previously detected parking lots. It was shown that by using all four cameras of a topview system, the recognition of parking lots of up to a distance of $5 \mathrm{~m}$ could be performed very reliably.

\section{Self-calibration of lens distortion from a mostly static scene}

In order to use video cameras for the localization of previously detected objects, an accurate calibration is necessary. It encompasses a precise model for lens distortion as well as the relative position and orientation of the camera with respect to the vehicle. The thesis presents procedures for an intrinsic camera self-calibration [6] while moving through a mostly static scene and demonstrates that lens distortion parameters can be estimated very accurately.

\section{Stereo vision in the area of overlap of two wide-angle cameras}

Another wide-spread application of video-based driver assistance is the computation of depth information from two or more cameras. The thesis maps out how the area of overlap from two or more cameras of a topview systems can be deployed to achieve this aim [8] (cf. Fig. 1b). Techniques to handle the large baseline and the strong distortions are presented. Experiments yield a range of up to $15 \mathrm{~m}$ for detecting vehicles.

\section{Self-localization in a parking scenario with known landmarks}

For ego-pose estimation in GNNS-denied environments, new approaches aim to detect landmarks whose position is stored within very accurate maps (cf. Fig. 1c). In this thesis, self-localization was achieved within an autonomous parking scenario with only a topview system using park markings as reference landmarks. After 
the prototype system was initialized with a coarse pose, it was possible to successively estimate the vehicle's trajectory through a roofed parking deck. The position estimation yielded an error below $10 \mathrm{~cm}$ and $2^{\circ}$.

\section{References}

[1] Leslie Lamport, A Document Preparation System, Addison-Wesley, 1994. (Example of Book Reference)

[2] I.T. Phillips, A.K. Chhabra, "Empirical performance evaluation of graphic recognition systems", IEEE Transactions on Pattern Analysis and Machine Intelligence 21(9):849-870, 1999. (Example of Journal Reference)

[3] H. Kida, O. Iwaki, K. Kawada, "Document recognition system for office automation”, IEEE Proc. of 8th Intl. Conf. on Pattern Recognition, Paris, 1:446-448, 1986. (Example of Proceedings Reference)

[4] Houben, S.: Bildverarbeitende Algorithmen zur Fahrerassistenz mit Weitwinkelkameras. / Image Processing Algorithms for Driver Assistance using Wide Angle Cameras (in German language). 2016 http://hssopus.ub.ruhr-uni-bochum.de/opus4/frontdoor/index/index/year/2016/docId/4520

[5] Caup, L. ; Salmen, J. ; Muharemovic, I. ; Houben, S.: Video-Based Trailer Articulation Estimation. In: Proceedings of the IEEE Intelligent Vehicles Symposium, 2013

[6] Houben, S.: Towards the Intrinsic Self-Calibration of a Vehicle-Mounted Omni-directional Radially Symmetric Camera. In: Proceedings of the IEEE Intelligent Vehicles Symposium Proceedings, 2014, S. 878-883

[7] Houben, S.: Park Marking Based Vehicle Self-Localization with a Fisheye Topview System. In: Journal of Real-Time Image Processing, 2015, DOI: 10.1007/s11554-015-0529-z

[8] Houben, S.: Topview Stereo: Combining Vehicle-Mounted Wide-Angle Cameras to a Distance Sensor Array. In: Proceedings of the IS\&T/SPIE Electronic Imaging Conference, 2015

[9] Houben, S. ; Komar, M. ; Hohm, A. ; Lüke, S. ; Neuhausen, M. ; Schlipsing, M.: On-Vehicle Video-Based Parking Lot Recognition with Fisheye Optics. In: Proceedings of the IEEE Conference on Intelligent Transportation Systems, 2013, S. 7 - 12 